\title{
Benign mucocele-like lesions of the breast: revisited
}

\author{
Shabnam Jaffer, Ira J Bleiweiss and Chandandeep S Nagi \\ Department of Pathology, The Mount Sinai Medical Center, New York, NY, USA
}

\begin{abstract}
Mucocele-like lesions of the breast are ruptured ducts that discharge their contents into the stroma. They constitute a spectrum from benign to atypical to malignant. The current management of these lesions diagnosed on core biopsy is excision. The goal of our study was to evaluate the necessity of this practice for benign mucocele-like lesions. Retrospective review of the pathology database from 1 January 2000 to 1 June 2008 identified 61 cases, with follow-up information available in 50 cases. Clinical, radiological, and pathological information was correlated. Core biopsies were reviewed to confirm the diagnosis and verify previous biopsy site. In all, 45 patients underwent surgery, whereas 5 patients were followed for $>1$ year and remained stable. Patient's ages ranged from 44 to 76 years. Most benign mucoceles were diagnosed stereotactically while targeting calcifications (93.3\%); rarely, the lesion was a sonographically detected mass. Most excisions had no residual mucocele $(37 / 45=82 \%)$. In seven cases $(15.6 \%)$, atypical duct hyperplasia was present, three with residual mucocele. In one case, the residual mucocele showed a continuum from florid to atypical duct hyperplasia at the core biopsy site. The other six cases showed atypical duct hyperplasia adjacent to but not directly at the core biopsy site. The sizes of the benign mucoceles ranged from incipient to $0.6 \mathrm{~cm}$, all containing calcifications except one, which was incidental. Radiological-pathological correlation was concordant in all cases except one with suspicious calcification, which was ductal carcinoma in situ on excision. In this series, the largest of its kind, the upstage rate of benign mucoceles diagnosed on core biopsy was $17.8 \%$. With the exception of the ductal carcinoma in situ case, no radiological or morphological features were predictive of atypia. Thus, because of associated atypical duct hyperplasia, sampling reasons, and intralesional heterogeneity, we continue to recommend excision of benign mucocele-like lesions diagnosed on core biopsy. Modern Pathology (2011) 24, 683-687; doi:10.1038/modpathol.2010.235; published online 14 January 2011
\end{abstract}

Keywords: benign mucocele-like lesion; core needle biopsy; excision

Historically, mucocele-like lesion of the breast was first described in 1986 by Rosen. ${ }^{1}$ He established the definition of mucocele-like lesions as 'mucin containing cysts lined by flat or low cubical epithelium with or without extravasated mucin'. Subsequent series found that mucocele-like lesions may be accompanied by a spectrum of pathological findings ranging from benign to atypical to malignant. ${ }^{2,3}$ In fact, in their series on benign and malignant mucocele-like lesions, Hamele-Bena et $a l^{2}$ found half of the cases associated with malignancy either in the form of intraductal carcinoma or invasive mucinous carcinoma. Thus, they recommended excisional biopsy for all mucocele-like lesions.

Correspondence: Dr S Jaffer, MD, Department of Pathology, Mount Sinai Hospital, 1 Gustave L. Levy Place, Box 1194, New York, NY 10029, USA.

E-mail: Shabnam.Jaffer@msnyuhealth.org

Received 25 August 2010; revised 2 November 2010; accepted 23

November 2010; published online 14 January 2011
These lesions were also felt to be precursors to mucinous carcinomas, given that mucocele-like lesion associated with atypia occurred a decade earlier than mucinous carcinoma and usually in the same region, when it preceded it.

It was also recognized that mucocele-like lesions were usually associated with calcifications and thus could be detected by mammography. With improved mammography, digital mammography, and the advent of image-directed biopsy specimens, most mucocele-like lesions are detected because of mammographic microcalcifications rather than a mass. A diagnosis of mucocele-like lesions on core biopsies is followed by a recommendation for excision because of the spectrum of lesions associated with mucocele-like lesions and sampling error. Although this practice may seem straightforward in cases with accompanying atypia or malignancy, the necessity of excising purely benign lesions diagnosed on core needle biopsy has been recently questioned. 
The few series that have addressed this issue have had mixed results with upstage rates ranging from 0 to $43 \%{ }^{4-9}$ Four of the six series ${ }^{4,6,8,9}$ showed atypia on excision, whereas two others showed no change between the core needle biopsy and excision findings of benign mucocele-like lesion. Based on their findings, the latter group has questioned the necessity of excising benign mucocele-like lesion diagnosed on core needle biopsy. With the exception of two recent series, ${ }^{8,9}$ none showed malignancy on excision, further justifying this practice. The goal of this study was to evaluate our experience and follow-up of benign mucocele-like lesions diagnosed on core needle biopsy.

\section{Materials and methods}

Using the pathology computer database of all core needle biopsy performed at our institution from 1 January 2000 to 1 June 2008, we identified 61/23 969 cases $(0.25 \%)$ of benign mucocele-like lesions, all biopsied by radiologists. All specimens were received fixed and processed routinely. Of the 61 patients, 45 underwent surgical excision, whereas 5 refused surgery and have remained stable for over 1 year. Also, 11 patients were lost to follow-up. All core biopsies were reviewed to confirm the diagnosis of benign mucocele-like lesion defined by the presence of mucin-containing dilated ducts that ruptured, with resultant extravasation of mucin into surrounding stroma (Figure 1). All excision specimens were reviewed to confirm the final diagnosis and document biopsy site changes. Clinical, radiological, and pathological correlation was performed.

\section{Results}

The age of the patients ranged from 44 to 76 years (mean $=54.6$ years). The method used to diagnose

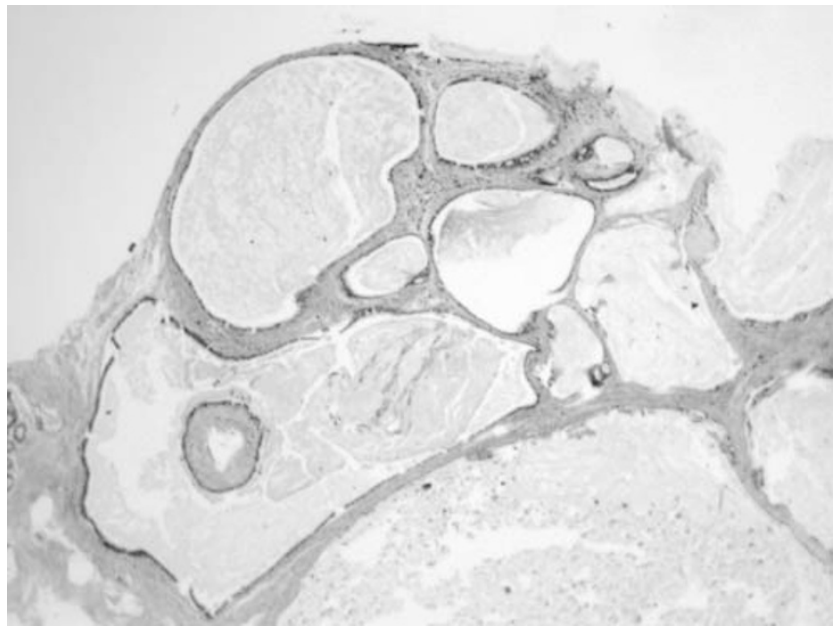

Figure 1 Benign mucocele-like lesion associated with calcifications diagnosed on core biopsy. benign mucocele-like lesion included stereotactic core needle biopsy using 11-14 gauge needle done for calcifications in 57 patients and ultrasound guided biopsy (USG) for a mass using 14-18 gauge needle in 4 patients. Systematic review of the imaging studies showed that the description of the calcifications was as follows: clustered $=37$, new $=10$, indeterminate $=3$, coarse $=3$, linear $=1$, granular $=1$, pleomorphic $=1$, and suspicious $=1$. On core biopsy, the benign mucocele-like lesion ranged in size from 0.1 to $0.6 \mathrm{~cm}$.

Biopsy site changes were identified in all patients who underwent excision (45; 42-stereotactic, 3ultrasound guided). A total of 37 patients had benign findings $(82 \%)$ such as fibrocystic changes with residual benign mucocele-like lesion in only 4 of the patients (Figure 2). In all, seven patients $(16 \%)$ had associated atypical duct hyperplasia, 3 with residual mucocele-like lesions (Figure 3).

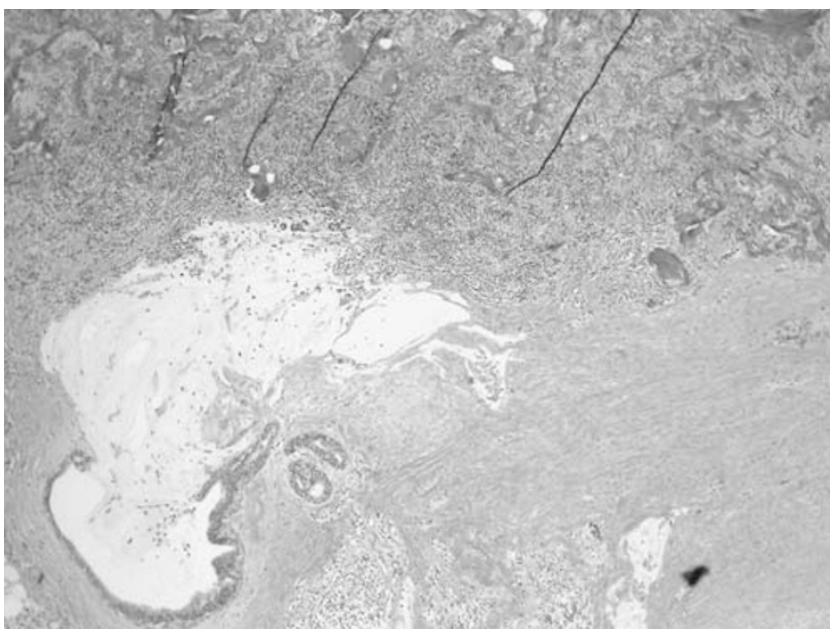

Figure 2 Residual benign mucocele-like lesion present at previous biopsy site changes.

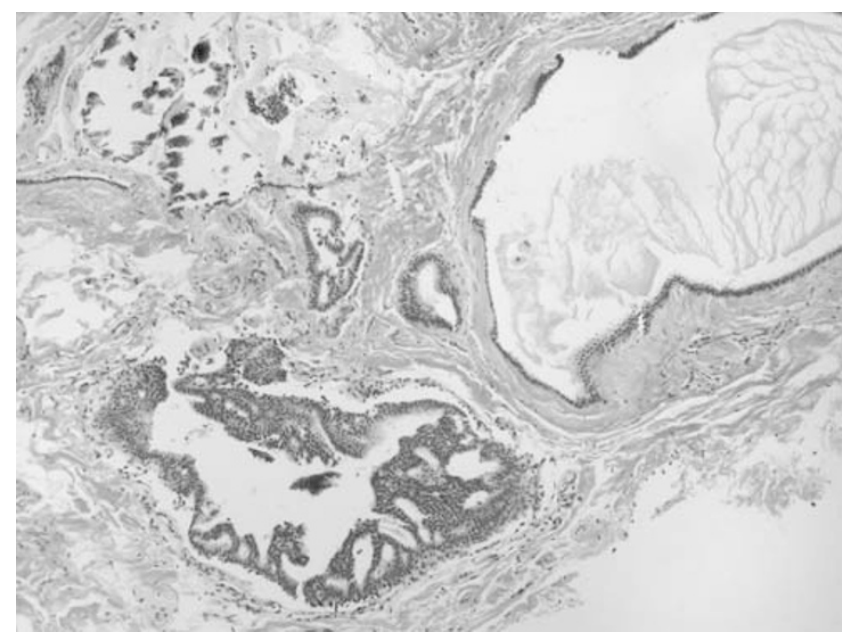

Figure 3 Excision specimen showing residual mucocele-like lesion associated with atypical duct hyperplasia and calcifications. 
Only 1 patient ( $2 \%$ ) was noted to have malignancy in the form of intraductal carcinoma associated with mucocele-like lesions. Thus, residual mucocele-like lesions was present in only 8 out of 45 cases $(18 \%)$ and ranged in size from 0.2 to $0.5 \mathrm{~cm}$.

We analyzed the clinical and morphological features of the cases with atypical duct hyperplasia and intraductal carcinoma and found that all of them were diagnosed stereotactically. Beginning with the atypical duct hyperplasia cases, with the exception of one case, the calcifications in all these cases were found to be present in the benign mucocele-like lesion on core biopsy (Figure 1). In the one exception case, the calcifications were present in fibrocystic changes and the benign mucocele-like lesion was incidental, measuring $0.1 \mathrm{~cm}$ (Figure 4). On excision, there was no residual mucocele-like lesion, but residual calcifications in atypical duct hyperplasia were identified. With the exception of two cases, the benign mucocele-like lesion ranged in size from 0.2 to $0.6 \mathrm{~cm}$. The two cases included: the incidental one already mentioned above and a second case that was incipient (Figure 5), that is, a mucin-filled cyst measuring $0.1 \mathrm{~cm}$ that had not yet ruptured. In the latter case, no residual mucocele-like lesions or calcifications were identified on excision, but there was atypical duct hyperplasia present adjacent to the biopsy site.

Residual calcifications were identified in five of the atypical duct hyperplasia cases. In three of these cases, no residual mucocele-like lesions were present, and the calcifications were present in atypical duct hyperplasia (two cases) and fibrocystic changes. In the remaining two cases with residual mucocele-like lesion, calcifications were identified in mucocele-like lesions in one case and both

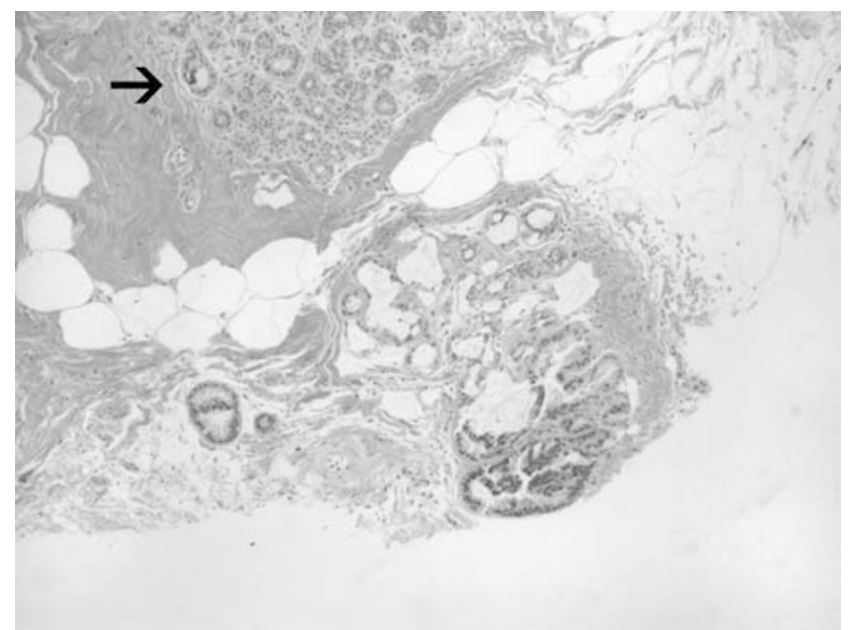

Figure 4 Incidental benign mucocele-like lesion characterized by minute size $(0.1 \mathrm{~cm})$ found on stereotactic core biopsy done for calcifications as seen exclusively in the fibrocystic changes (see arrow). atypical duct hyperplasia and mucocele-like lesions in the other case. We next studied the relationship of the biopsy site to the pathology and found only one case of atypical duct hyperplasia at the biopsy site, continuous with an area of mucocele-like lesions with florid duct hyperplasia. In the remaining six cases, the atypical duct hyperplasia was adjacent to, but not at the biopsy site.

The indication for biopsy in the intraductal carcinoma case was suspicious and widespread calcifications. On core biopsy, the benign mucocele-like lesion measured $0.3 \mathrm{~cm}$ and was associated with calcifications (Figure 6), but did not entirely correlate with the finding of suspicious calcifications. There was also adjacent atypical lobular hyperplasia (Figure 6). The excision specimen showed residual mucocele-like lesions with atypical duct hyperplasia and lobular carcinoma in situ admixed with focal low-grade intraductal

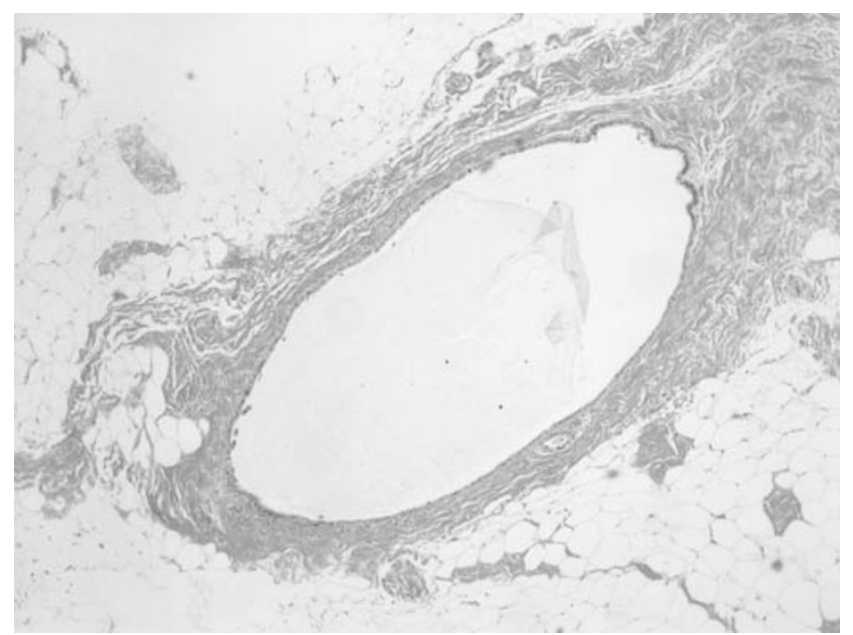

Figure 5 Incipient mucocele-like lesion.

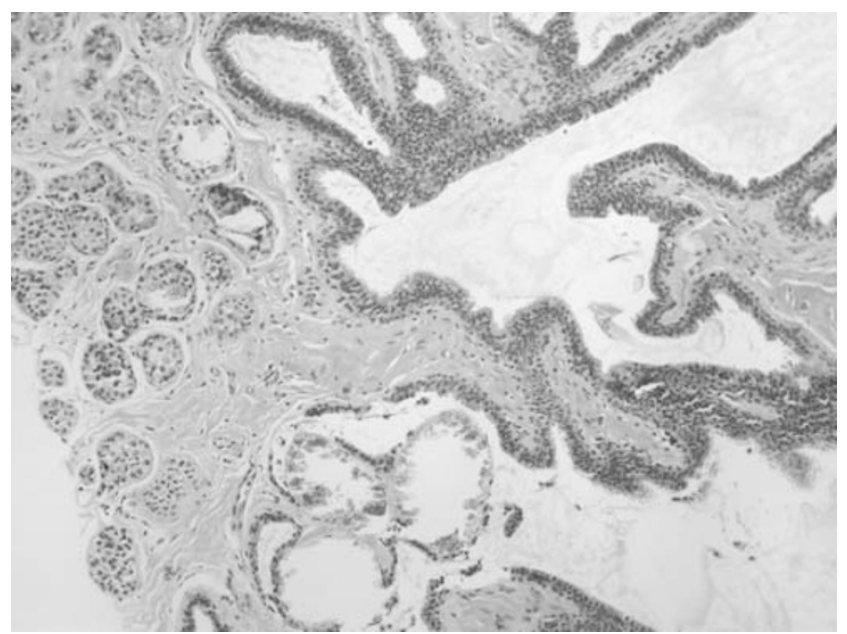

Figure 6 Core biopsy showing benign mucocele-like lesion with adjacent atypical lobular hyperplasia, both associated with calcifications. 

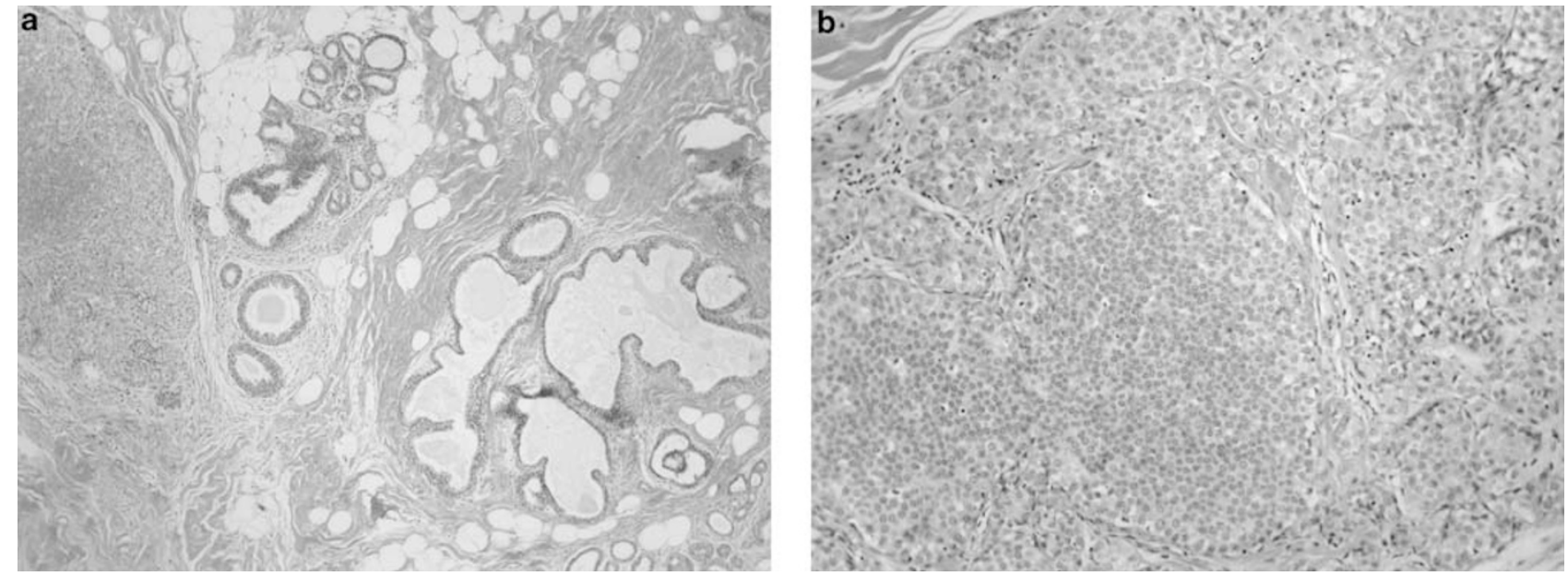

Figure 7 Excision specimen of Figure 6 showing residual mucocele-like lesion with adjacent in situ carcinoma (a). High power shows mixed ductal and lobular features (b).

carcinoma associated with mucin and calcifications (Figure 7).

\section{Discussion}

The early series describing mucocele-like lesions were primarily in specimens that consisted of excisional biopsies, lumpectomies, and mastectomies. These studies provided evidence that a morphological spectrum existed from benign to atypical to malignant, and thus complete excision of these lesions was recommended. ${ }^{1,2}$ With the advent of core needle biopsies, the practice of excising mucocele-like lesions was similarly continued primarily because of issues related to sampling a heterogeneous lesion. Although the diagnosis of mucocele-like lesions proved challenging on limited fragmented core biopsy material, it was nevertheless feasible as proven by Renshaw, ${ }^{4}$ although in a small series consisting of only 22 cases. In his series, although there was no misdiagnosis of any benign cases to malignancy or vice versa, one of three (33\%) excised benign mucocelelike lesions proved to have atypical duct hyperplasia on excision. Similarly, Wang et $a l^{6}$ showed in their series that the majority of mucinous lesion can be accurately diagnosed by core biopsy. Given that all their benign mucocele-like lesions remained benign on excision, they questioned the necessity of surgical excision. On the other hand, Carder et al ${ }^{5}$ showed an upstage of benign mucocele-like lesion to atypical duct hyperplasia in three out of seven cases $(43 \%)$ from core biopsy to excision. Based on their results, they advocated excision on mucocele-like lesions diagnosed on core biopsies, even benign mucocele-like lesion because of the associated risk of atypical duct hyperplasia on excision. In the excisions of benign mucocele-like lesion diagnosed on core in Nguyen et al, ${ }^{7}$ there was no upstaging to atypia or malignancy. Thus, it was felt that follow- up alone was appropriate for benign mucocele-like lesion; however, this series was limited by only six cases that were excised. On the other hand, in the study of Phillips et $a l^{8}$ of seven benign mucocelelike lesions that were excised, one was upstaged to atypical duct hyperplasia and another to intraductal carcinoma. Thus, this group recommended excision of all benign mucocele-like lesions, particularly if they presented as a mass. Similarly, in the most recent study of mucocele-like lesions by Begum et $a l,{ }^{9}$ two out of 39 cases of benign mucocele-like lesions were upstaged; one was upstaged to colloid carcinoma and had been composed of acellular mucin pools on core biopsy, whereas the other case's excision showed atypical duct hyperplasia. Based on this study, the authors felt that if the entire radiological lesion was completely removed by a large core biopsy, particularly calcifications, surgery could be avoided. However, they cautioned that radiological-pathological correlation and further validation of these findings in larger studies is necessary. Thus, as can be seen by the results of these series, there are varying opinions and no true consensus regarding the management of benign mucocele-like lesion diagnosed on core biopsy.

To attempt to resolve this issue, we looked at our study composed of 61 benign mucocele-like lesions, the largest of its kind. We found that benign mucocele-like lesion tends to occur in older women, usually detected because of clustered calcifications rather than a mass. Our upstage rate was $18 \%$, compared with $0-43 \%$ in the literature as described above. In our series, it was primarily because of atypical duct hyperplasia (88\%), with only one case of intraductal carcinoma, all diagnosed stereotactically. Despite the low upstage to malignancy on excision, a mere $2 \%$, we would still recommend excision of mucocele-like lesion because of the $16 \%$ associated risk of atypical duct hyperplasia. This is because it is hypothetically recognized that atypical duct hyperplasia and intraductal carcinoma are part 
of an evolving spectrum. Excising atypical duct hyperplasia is akin to excising the precursor lesion to malignancy, thus preventing the theoretical progression to malignancy. In fact, a diagnosis of atypical duct hyperplasia is an important factor in the Breast Cancer Risk Assessment Tool (the Gail model). Additionally, the diagnosis of atypical duct hyperplasia confers a four- to five-fold increased risk over that of the general population for the development of malignancy in either breast. Consequently, patients with a diagnosis of atypical duct hyperplasia are offered more frequent screening and some, depending on their risk factor index, are also offered antihormonal treatment such as tamoxifen. Thus, the upgrade from mucocele-like lesion on core biopsy to atypical duct hyperplasia may not be as significant as malignancy but, nevertheless, has relevant clinical implications.

Most mucocele-like lesions were removed by the core biopsy with no appreciated residual component on excision $(82 \%)$. Unlike other studies, we did not find the biopsy needle gauge size, cellularity of the mucocele-like lesions, residual mucocele-like lesions or calcifications, size of the mucocele-like lesions, or whether it presented as a mass or calcifications as being predictive of residual mucocele-like lesions, atypia, or malignancy. In fact, with regard to size, in two of our cases upstaged to atypical duct hyperplasia, the mucocele-like lesions were incidental and incipient, measuring $<1 \mathrm{~mm}$.

In the atypical duct hyperplasia cases, the finding of atypical duct hyperplasia close to but not at the biopsy site in most cases suggests an association between mucocele-like lesions and atypia. Although some have advocated the use of large gauge needles as an alternative to excision of benign mucocele-like lesion provided radiological-pathological correlation is performed, our series shows the contrary. Both incidental and incipient mucocele-like lesions that were entirely removed by core needle biopsy were found to have associated atypical duct hyperplasia on excision. This then justifies the practice of excising the area immediately contiguous to the benign mucocele-like lesion because of associated atypia, despite no residual mucocele-like lesions or even calcifications.

The single intraductal carcinoma case stresses the necessity of radiological-pathological correlation in all these cases. Given the discordance between the presence of mammographically suspicious calcifications and the finding of benign mucocele-like lesion with calcifications, excision was done. Residual suspicious calcifications were present. Excision of these calcifications showed a spectrum of findings from residual mucocele-like lesions with atypical duct hyperplasia, lobular carcinoma in situ, and intraductal carcinoma. Thus, discordance between radiology and pathology must always be resolved by excision.

\section{Conclusions}

The excision of benign mucocele-like lesion has been questioned by some. In our series, although most benign mucocele-like lesions were removed by the core biopsy, a residual component was present in $\sim 18 \%$ of cases and an upstage occurred in $18 \%$. In terms of malignancy, we only had one case of intraductal carcinoma, which was because of radiological-pathological discordance. We agree with previous studies that it is prudent to continue to excise all benign mucocele-like lesions diagnosed on core needle biopsy because of sampling phenomena, intralesional heterogeneity, and associated atypia or malignancy.

\section{Disclosure/conflict of interest}

The authors declare no conflict of interest.

\section{References}

1 Rosen PP. Mucocele-like tumour of the breast. Am J Surg Pathol 1986;10:464-469.

2 Hamele-Bena D, Cranor ML, Rosen PP. Mammary mucocele-like lesions. Benign and malignant. J Surg Pathol 1996;20:1081-1085.

3 Weaver MG, Abdul-Karim FW, Al-Kaisi N. Mucinous lesion of the breast: a pathological continuum. Pathol Res Pract 1993;189:873-876.

4 Renshaw A. Can mucinous lesions of the breast be reliably diagnosed by core needle biopsy? Am J Clin Pathol 2002;118:82-84.

5 Carder PJ, Murphy CE, Liston JC. Surgical excision is warranted following a core biopsy diagnosis of mucocele-like lesion of the breast. Histopathology 2004;45: 148-154.

6 Wang J, Simsir A, Mercado C, et al. Can core biopsy reliably diagnose mucinous lesions of the breast? Am J Clin Pathol 2007;127:124-127.

7 Nguyen CV, Resetkova E, Huo L. Mucocele-like lesions of breast with no or minimal epithelial atypia on core biopsy: to excise or not? Mod Pathol 2009;22(S1): 59A-60A.

8 Phillips DGK, Sailey CJ, Warner J, et al. Mucocele-like lesions diagnosed by core needle biopsy of the breast: correlation with surgical excision. Mod Path 2009;22(S1):63A.

9 Begum SMKN, Jara-Lazaro AR, Thike AA, et al. Mucin extravasation in breast core biopsies - clinical significance and outcome correlation. Histopathology 2009;55: 609-617. 